\title{
ERRATUM TO: CONTINUOUS ORTHOGONAL COMPLEMENT FUNCTIONS AND DISTRIBUTION-FREE GOODNESS OF FIT TESTS IN MOMENT STRUCTURE ANALYSIS
}

ROBERT JENNRICH

UNIVERSITY OF CALIFORNIA, LOS ANGELES

ALBERT SATORRA

UNIVERSITAT POMPEU FABRA, BARCELONA

\section{Erratum to: PSYCHOMETRIKA \\ DOI 10.1007/s11336-013-9320-x}

On pages 5th and 6th of the manuscript

1. Page 5th,

(a) at line -17 , an " $n$ " is missing; needs to be

$$
T_{n}=n r_{n}^{\prime} U_{n}\left(U_{n}^{\prime} \Gamma_{n} U_{n}\right)^{-1} U_{n}^{\prime} r_{n} \stackrel{D}{\rightarrow} \chi_{p-q}^{2} ;
$$

(b) in equality (5), an " $\sqrt{n}$ " is missing; needs to be

$$
V_{n}^{\prime} \sqrt{n} r_{n} \stackrel{a}{=} V^{\prime} \sqrt{n}\left(s_{n}-\sigma_{0}\right) \stackrel{D}{\rightarrow} \mathcal{N}\left(0, V^{\prime} \Gamma V\right) ;
$$

(c) at line -3 , an " $n$ " is missing; needs to be

$$
S_{n}=n r_{n}^{\prime} V_{n}\left(V_{n}^{\prime} \Gamma_{n} V_{n}\right)^{-1} V_{n}^{\prime} r_{n} \stackrel{D}{\rightarrow} \chi_{p-q}^{2} .
$$

2. Page 6th, at lines 1 to 3 , an " $n$ " is missing in each line; needs to be

$$
\begin{aligned}
T_{n} & =n r_{n}^{\prime} U_{n}\left(U_{n}^{\prime} \Gamma_{n} U_{n}\right)^{-1} U_{n}^{\prime} r_{n} \\
& =n r_{n}^{\prime} V_{n} M_{n}\left(M_{n}^{\prime} V_{n}^{\prime} \Gamma_{n} V_{n} M_{n}\right)^{-1} M_{n}^{\prime} V_{n}^{\prime} r_{n} \\
& =n r_{n}^{\prime} V_{n}\left(V_{n}^{\prime} \Gamma_{n} V_{n}\right)^{-1} V_{n}^{\prime} r_{n} .
\end{aligned}
$$

Published Online Date: 25 APR 2013

The online version of the original article can be found under doi:10.1007/s11336-013-9320-x.

Requests for reprints should be sent to Robert Jennrich, University of California, Los Angeles, 3400 Purdue Ave., Los Angeles, CA, USA. E-mail: rij@stat.ucla.edu 